\title{
Estrategias de integración sensorial en la educación infantil
}

\author{
Strategies for sensory integration in early childhood education \\ Yolanda Patricia Castellanos Mora* y \\ Martha Esperanza Melo Tinjacá**
}

\section{RESUMEN}

El propósito de este artículo es destacar la importancia de las es-

Palabras clave: trategias de integración sensorial en el aula de clase, para favoreintegración cer las necesidades del desarrollo integral de niños y niñas y sus procesos neurológicos, los cuales le permiten asimilar, procesar e interpretar la información sensorial recibida para dar una respuesta adecuada. Para ello, se planteó la realización de talleres lúdicos, teniendo en cuenta conceptos y herramientas que se trabajan en la teoría de integración sensorial. La investigación se sensorial, desarrollo integral, sistema táctil, sistema vestibular y sistema propioceptivo. realizó con una población de 40 estudiantes, entre los 4 y 6 años, empleando una metodología mixta - tomando lo cuantitativo en la recogida de datos estadísticos y lo cualitativo en la observación realizada en los talleres-. Se aplicó un pretest y un postest, correspondientes a la escala abreviada de desarrollo, y 10 talleres de intervención de integración sensorial, detectándose la necesidad de fortalecimiento en el desarrollo de los procesos sensoriales. Se concluye que los talleres fueron de gran motivación para los estudiantes, los cuales respondieron adecuadamente a los estímulos: en el sistema táctil con un 95\%, en el sistema propioceptivo con un $80 \%$, y en el sistema vestibular con un $89 \%$. De igual manera, se detectaron dificultades en algunos estudiantes, a los cuales se seguirá prestando apoyo.

Estudiante de Maestría en Educación, cuarto semestre Fundación Universitaria Los Libertadores. Especialista en Pedagogía de la Lúdica para el Desarrollo Cultural. Licenciada en Educación Preescolar. Docente de Preescolar en la Escuela Normal Superior Distrital María Montessori. Contacto: patriciacm@gmail.com

* $\quad$ Estudiante de Maestría en Educación, cuarto semestre Fundación Universitaria Los Libertadores. Licenciada en Pedagogía Infantil. Docente de Preescolar del Jardín Infantil El Globito Rojo. Contacto: memelot@libertadores.edu.co 


\section{SUMMARY}

This article's objective is to highlight the importance of sensory integration strategies in the classroom to encourage the needs of children's integral development and their neurological processes, allowing them to assimilate and interpret the sensory information received to give an appropriate response. It was proposed to hold playful workshops, taking into account concepts and tools from the sensory integration theory. The study used a sample of 40 students, between the ages of 4 and 6 years old, using a mixed methodology - considering statistical data collective as quantitative and workshop observation as qualitative. A pre-test and a post-test were applied, corresponding to the abbreviated scale of development, and ten intervention workshops of sensory integration, detecting the need for strengthening the development of sensory processes. It was concluded that workshops motivated students, who responded adequately to the stimuli: tactile system with $95 \%$, proprioception system with $80 \%$, and in the vestibular system with $89 \%$. Similarly, difficulties were detected in some students, who will continue to be supported.
Key words: sensory integration, integral development, tactile system, vestibular system, and proprioceptive system. 


\section{Introducción}

Uno de los propósitos más importantes de la educación inicial es potenciar el desarrollo de niños y niñas durante esta etapa. Para esto, dentro del trabajo pedagógico está el ofrecer experiencias desafiantes, que impulsen el desarrollo mediante el juego y la exploración del medio, entre otras incluidas en las políticas que reglamentan la educación inicial en Colombia (Decreto 1075, Ministerio de Educación Nacional, 2015).

Por este motivo, todas las acciones que se realizan en el aula de clase permiten potenciar lo que el niño sabe hacer y lo que puede aprender para lograr un mejor desarrollo integral.

Es preponderante el empleo de estrategias educativas sensoriales primordialmente en la etapa infantil, lapso de apogeo del desarrollo psicomotor, en el que el volumen e integración de información sensitiva es crítica, sumado a la curiosidad y el placer que el niño o la niña extraen de la experiencia sensorial. (Camacaro, 2013, p. 93).

La presente investigación es de carácter académico y su principal motivación es contribuir en la innovación de los espacios pedagógicos de la primera infancia para el fortalecimiento, desarrollo y transformación, reconociendo que una educación de calidad en niñas y niños significa aportar y ampliar las posibilidades y progresos en el desarrollo sensorial, para favorecer las rutinas que se vivencian en la escuela. Lucy Jane Miller, en su libro No longer a secret, afirma que los docentes son la columna vertebral del futuro de un país y que la tarea del buen docente es estimular a las personas comunes y corrientes a un esfuerzo inusual (Miller \& Bialer, 2012).

Emplear estrategias para trabajar la integración sensorial en clase es generar una diversión y juego a manera de aprendizaje, lo cual motiva al niño, ya que es su modo natural de aprender y un pilar fundamental en la primera infancia. En cada actividad planteada en la propuesta se busca proveer al niño de variadas sensaciones, las cuales se convierten en nutrición para el cerebro, "buscando de manera natural, las sensaciones que ayudan a organizar nuestro cerebro. Esta es una de las razones por las que los niños les encanta ser cargados, acunados, abrazados, y por la que les gusta correr, saltar y jugar" (Ayres, 2006, p. 16). 
Muchas veces se observan, en el aula de clases, niños con diversas dificultades que pueden estar afectando su desarrollo, por lo que los maestros buscan diversas formas de apoyo. La estrategia propuesta en esta investigación contribuye al desarrollo de la autorregulación, el bienestar, la adquisición de destreza y planeamiento motriz, y la disposición para aprender, lo que se traducirá en personas más capaces de enfrentar los retos que la vida diaria y académica les presentan (Yack, Sutton \& Paula, 2002).

En el tema de la integración sensorial (IS) hay cada vez más investigaciones y recursos online que buscan favorecer la educación de los estudiantes, entendiendo que cada niño y niña puede tener algunas dificultades en ciertos aspectos y que se puede favorecer a todos como lo establecen las políticas de inclusión. Los educadores deben estar cada vez más documentados para abordar de manera adecuada las diferentes necesidades sensoriales individuales de niños y niñas, haciendo cambios en las prácticas pedagógicas y buscando soluciones a los nuevos desafíos que se enfrentan (Senkow, 2018).

\section{La integración sensorial}

La integración sensorial (IS) es un proceso neurobiológico referido a la habilidad para organizar la información sensorial, para su propia aplicación y para responder apropiadamente en el ambiente. El sistema nervioso central (SNC) se encarga de procesar, interpretar e igualmente generar respuestas ante los estímulos sensoriales. La teoría de IS, desarrollada por Jean Ayres (1920-1988), terapeuta ocupacional y doctora en psicología de la educación (Ayres, 1998), afirma que:

La integración sensorial es la organización de sensaciones para su uso, y fluyen al cerebro como arroyos a un lago. Nuestros sentidos nos dan la información acerca de las condiciones físicas de nuestro cuerpo y del ambiente que nos rodea, incontables trozos de información entran a nuestro cerebro, no sólo por nuestros ojos y oídos sino también de cada punto de nuestro cuerpo. Poseemos un sentido especial que detecta la fuerza de gravedad y los movimientos de nuestro cuerpo en relación con la tierra. (p. 13-14).

Desde la gestación se inicia el desarrollo de la integración sensorial; igualmente, tan pronto el bebe nace, tendrá diversas experiencias 
sensoriales. El sentido auditivo y el táctil son los que primero se desarrollan, y permiten al niño aprendizajes iniciales en los cuales empieza a dar respuestas adaptativas de aquellas sensaciones vivenciadas: "un niño pequeño no tiene muchos pensamientos o ideas sobre las cosas, únicamente se ocupa de sentirlas y mover su cuerpo en relación con esas sensaciones" (Ayres, 2006, p. 17). De esta manera, en la primera infancia el cerebro se ocupa de recibir información a través de los sentidos. De tal forma, gracias a la integración sensorial, el bebé empieza a realizar diversos movimientos y podemos ver cómo aprende a gatear, a intentar mantenerse en pie y a caminar.

Los sistemas sensoriales, por tanto, se inician tempranamente:

Desde la semana cinco de la gestación aproximadamente y hasta el séptimo año de vida se da la maduración de los sistemas sensoriales, permitiéndole al niño desarrollar habilidades sensorio-motrices, cognitivas e intelectuales que servirán de base para el óptimo desempeño del juego, las actividades escolares y las actividades básicas de la vida diaria. (Alves, 2017, p. 10).

Según Jean Ayres, la integración sensorial puede producir cambios en el cerebro, esto gracias a la cantidad de estimulaciones sensoriales, vestibulares y táctiles que se proveen al niño en diferentes momentos de su vida. El cerebro tiene la cualidad de ser maleable, y con mayores posibilidades el cerebro joven, lo cual repercute en estructuras y funciones más firmes con el pasar de los años. Es así como durante la primera infancia se hace necesaria la adecuada estimulación, ya que se cuenta con un mapa mental dinámico y plástico, denominándose esta etapa como "periodos sensibles" o "ventanas de oportunidad", establecidas hasta los ocho años, según los estudios (Díaz, 2013).

Diferentes investigaciones afirman que el desarrollo sensorial va ligado al desarrollo cognitivo, ya que todo lo que se percibe llega al cerebro a través de los sentidos, el que se encarga de procesar la información, interpretarla y dar una respuesta adecuada. Por esto "es muy importante cuidar, conocer y fortalecer el desarrollo de las sensaciones en el niño, ya que estas serán la base sobre la cual se formarán los procesos superiores de conocimiento, inteligencia y lenguaje" (Navas, 2011). Si bien el desarrollo normal de la integración sensorial se ubica entre los tres y los siete años de vida, gracias a la plasticidad de las 
neuronas o neuroplasticidad del cerebro, aun las personas no estén en este rango de edad y presenten dificultades de aprendizaje, pueden presentar cambios significativos con diversas estrategias o, cuando sea pertinente, con el apoyo de terapias de integración sensorial (Beaudry, 2003).

Según Carol Stock, un buen desarrollo de la integración sensorial se resume en un buen empleo de los sentidos, utilizando estos eficaz y simultáneamente (Stock, 1998, p. 18). Así, cuando este proceso de interpretación establece respuestas adecuadas, el niño puede enfrentarse de mejor manera a las demandas que el entorno y la cotidianidad le presentan, tanto en actividades rutinarias como el ambiente social y escolar.

Según Bialer y Miller (2011), por medio de los sistemas sensoriales del cuerpo se recibe información o input sensorial que es interpretada por el cerebro. Cada sensación es diferente en cada persona, de acuerdo con las variadas interpretaciones que se da a esta información o las asociaciones que llegue a tener con las experiencias sensoriales. En la teoría de integración sensorial están incluidos los cinco sentidos que se conocen tradicionalmente (vista, audición, tácto, gusto y olfato), y dos más incluidos en la literatura de IS: vestibular (fuerza de gravedad y movimiento de la cabeza) y propioceptivo (posición y movimiento de músculos y articulaciones).

El sistema sensorial permite el acercamiento al mundo que nos rodea. Estos órganos especializados localizan los estímulos para que luego esta información pase al SNC, entonces éste se encarga de organizar la información. Los sistemas de integración sensorial más primitivos y que maduran de primero son el táctil, vestibular y propioceptivo; tienen una gran influencia en cómo se responde a la información visual y auditiva, y son aquellos en los que se enfoca principalmente la teoría de la integración sensorial.

Cada uno de los sistemas sensoriales aporta al desarrollo del niño, de acuerdo con Erma Blanche (1998), quien afirma que cada uno de los sistemas sensoriales realiza contribuciones importantes en el proceso sensorial, como se describe en la siguiente tabla. 
Tabla 1.

\begin{tabular}{|c|c|}
\hline \multicolumn{2}{|c|}{ Contribuciones de los sistemas sensoriales al desarrollo } \\
\hline Sistema sensorial & Desarrollo \\
\hline Táctil & $\begin{array}{l}\text { - Reflejos primitivos: búsqueda, succión y prensión } \\
\text { - Desarrollo emocional: apego y vinculación } \\
\text { - Esquema corporal y oral-motor } \\
\text { - Protección ante estímulos potencialmente dañinos }\end{array}$ \\
\hline Vestibular & $\begin{array}{l}\text { - Seguridad emocional en función de la gravedad } \\
\text { - Reflejos y respuestas motoras en función de la } \\
\text { gravedad, extensión contra gravedad, reacciones } \\
\text { de equilibrio, control postural y mantenimiento de } \\
\text { posturas contra gravedad, y mantener la cabeza en } \\
\text { posición vertical } \\
\text { - Manejo del cuerpo en el espacio: orientación } \\
\text { espacial, mantener un campo visual estable, } \\
\text { coordinación motora bilateral, anticipación (cómo } \\
\text { moverse en el espacio en diferentes situaciones) } \\
\text { - Trabajo conjunto con el sistema propioceptivo }\end{array}$ \\
\hline Propioceptivo & $\begin{array}{l}\text { - Reflejos que median la alineación del tronco, cabeza } \\
\text { y otras partes del cuerpo } \\
\text { - Modulación del movimiento: control del rango de } \\
\text { amplitud del movimiento y fluidez y precisión del } \\
\text { movimiento de las diferentes partes del cuerpo }\end{array}$ \\
\hline Gustativo/olfativo & $\begin{array}{l}\text { - Vínculo con el cuidador } \\
\text { - Protección frente a sustancias nocivas } \\
\text { - Atracción a sustancias nutricionales de crianza }\end{array}$ \\
\hline Visual & $\begin{array}{l}\text { - Vínculo con el cuidador: emergencia del contacto } \\
\text { visual, agudeza visual y percepción de la distancia } \\
\text { - Informa al individuo sobre la distancia de los } \\
\text { estímulos presentes en el medio } \\
\text { - Orientación espacial } \\
\text { - Crítico en las funciones sociales de comunicación } \\
\text { compleja }\end{array}$ \\
\hline Auditivo & $\begin{array}{l}\text { - Informa al individuo sobre la distancia de los } \\
\text { estímulos presentes en el medio } \\
\text { - Orientación espacial } \\
\text { - Crítico en el desarrollo del lenguaje }\end{array}$ \\
\hline
\end{tabular}

Fuente: Ramírez, 2016.

Es importante entender que todas las personas desarrollamos procesos de integración sensorial; sin embargo, mientras en unos se desarrolla adecuadamente, otros, en menor o mayor grado, presentan algunas insuficiencias traducidas en dificultades que interfieren en muchas situaciones de la vida, ya que habrá más esfuerzo que causará 
problemas de aprendizaje y de comportamiento, por todo lo que en ella interfiere, debido a que el cerebro no está organizando las sensaciones de manera correcta.

Isabelle Beaudry, terapeuta ocupacional y directora de la Clínica de Terapia Ocupacional pediátrica Beaudry-Bellefeuille, de Oviedo, España, desde hace años desarrolla investigaciones y literatura dirigida no solo a terapeutas sino también a las familias y docentes.

Entre sus libros están: Tengo duendes en las piernas, Problemas de aprendizaje en la infancia y Hago lo que veo, soy lo que hago. A través de ellos, busca informar, en un lenguaje sencillo, respecto de la importancia del proceso y del desarrollo de integración sensorial desde la infancia, enseñando pautas y actividades que potencien el procesamiento, la organización y las respuestas del cerebro para favorecer la vida y el aprendizaje del niño. Ya que es un tema poco conocido entre profesores y padres de familia, es importante entender que, al procesar la información, muchos niños, jóvenes y adultos aún presentan dificultades.

\section{Sentido táctil}

El sistema táctil es el sistema más amplio y tiene la cualidad de trabajar eficazmente cuando los sistemas visual y auditivo todavía no están totalmente desarrollados, su función es recibir la información de la piel, la cual cuenta con receptores que detectan variadas sensaciones. Este sistema cuenta con dos subsistemas para la percepción, que son el de protección y el discriminativo; el primero ayuda a dar una respuesta a un estímulo nocivo y el discriminativo incorpora con detalle las características de las cosas externas, discriminando objetos suaves, lisos, ásperos, calientes, fríos, entre otros.

La teoría de integración sensorial se centra sobre todo en el sistema táctil. Según Gonzáles, 2006, citado en Camacaro, 2013), el tacto es la modalidad sensorial que ofrece al cerebro información sobre las propiedades de los cuerpos. Este sentido hace posible una experiencia palpable y corpórea, mientras que la visión, la audición y el resto aportan percepciones inmateriales e intangibles.

El sentido táctil permite, desde edades tempranas, diferentes percepciones que permiten aprender conceptos; estas dependen de la cantidad de experiencias que se brinden a niños y niñas. Dentro de 
estas percepciones podemos nombrar "los diferentes tipos de superficies, consistencia, temperatura, peso y dimensiones de los cuerpos, formas de lo que tocamos, grados de humedad o sequedad, facilidad o dificultad de su uso" (García, 1996, p. 214).

En un nivel táctil, niños y niñas pueden presentar hipersensibilidad ante diferentes texturas, algunos materiales o incluso comidas. Esto causa graves molestias, las cuales no son comprendidas por los adultos $\mathrm{y}$, en algunas oportunidades, pueden ser mal interpretadas, lo que se traduce en reacciones de rechazo e incomprensión. También sucede la llamada "defensividad táctil", en la que, al ser tocado por otra persona, el infante reacciona con malestar y rechazo.

\section{Sistema vestibular}

Es uno de los sistemas fundamentales en la teoría de integración sensorial y se encuentra ubicado en el oído interno, en una estructura denominada "laberinto". Provee la información sensorial de la posición de la cabeza asociada con la gravedad, y reconoce qué movimientos están sucediendo y si nos movemos hacia arriba o hacia abajo. Igualmente, responde a sensaciones de la cabeza, como aceleración o desaceleración, y movimientos lentos o suaves. Cuando las personas tienen dificultades con este sistema suelen sentir nauseas o mareos (Bialer \& Miller, 2011, p. 24).

En los niños de preescolar que presentan dificultades en un nivel vestibular se identifican conductas como caerse con frecuencia del puesto, falta de coordinación, movimientos impulsivos y riesgosos, como vueltas o saltos poco controlados; también, manifiestan cansancio físico con mucha facilidad, necesitan apoyarse en algún compañero constantemente, tienen dificultades para mantener una adecuada postura y para centrar la atención, además de inconvenientes en escritura. En otros casos pueden mostrar temor a las sensaciones de movimiento, a bajar escaleras, y evitan las experiencias en clases de educación física o en la dimensión corporal motora gruesa.

Estas deficiencias vestibulares empiezan a repercutir en la identidad del niño, ya que, al ver que no se le facilitan desarrollos motores gruesos, se compara son sus compañeros que sí cuentan con ciertas habilidades, y esto empieza a afectar su concepto de sí mismo: siente que no tiene los talentos o que es malo en su desempeño. 
Por tanto, se hace necesario que, desde la escuela, se realicen intervenciones para favorecer el sistema vestibular. Al respecto, Alfonso Lázaro afirma que en educación inicial se puede realizar actividades que involucren equilibrio con diferentes elementos, como balones, zancos, plataformas con ruedas (scooter), entre otros, ya que permiten un mejor desarrollo. Entre estas nociones el autor incluye la "educación de las sensaciones plantares, adaptación a la altura, educación de la caída, balance y giros, aceleraciones rectilíneas, angulares y verticales" (Lazáro, 2008, p. 172).

\section{Sistema propioceptivo}

Provee información de la posición de las partes del cuerpo, de la extensión y contracción de los músculos tendones y las articulaciones. Ofrece "una impresión del estado de movimiento, relajación o equilibrio de nuestro cuerpo" (Martínez, 2014). Igualmente, se relaciona estrechamente con el esquema corporal, el equilibrio, la coordinación motora y la modulación de niveles de alerta. En este sistema se describen términos como la "sensibilidad propioceptiva", es decir, la que informa acerca de la postura corporal (forma de colocar el cuerpo en el espacio) y de las articulaciones; otro término es el "tono muscular", que se refiere al grado de tensión de los músculos (Sanchez, 2010).

Uno de los aspectos importantes en el desarrollo infantil es el "esquema corporal", en el cual se incluyen tres estadios; estos son, según Ajuriaguerra (1969), el nivel del cuerpo vivido (el niño empieza a tomar conciencia de su propio cuerpo); el nivel del cuerpo percibido (puede reconocer y nombrar algunas partes de su cuerpo), y el nivel cuerpo representado (logra representar su cuerpo) (Martínez, 2014). En las intervenciones para favorecer el desarrollo del sistema propioceptivo se ofrecen experiencias sensoriales de modulación, que incluyen nivel de alerta, atención, organización, modificaciones ambientales y actividades recreativas. También se incluyen las denominadas "respuestas adaptativas", que involucran coordinación motora, planteamiento motor y control postural.

\section{Desarrollo de la integración sensorial}

El proceso de la IS, según Jean Ayres, propone un desarrollo secuencial del niño por niveles, en el que las habilidades adquiridas en cada etapa 
proporcionan las bases para el desarrollo de comportamientos cada vez más complejos. En el primer nivel, las experiencias táctiles, vestibulares y propioceptivas son las de mayor influencia; en el segundo el niño desarrolla su imagen corporal y planifica mejor sus movimientos; en el tercer nivel el niño logra un desarrollo mayor del habla y del lenguaje, de la coordinación ojo-mano, de la percepción de forma y espacio, y de la actividad con meta; en el cuarto nivel logra habilidades para concentrarse, organizarse, desarrollar control de sí mismo adquiriendo confianza en sus medios.

En el desarrollo de la IS se presentan cuatro niveles. En el primero ocurre la postura y el equilibrio, tono muscular, seguridad en el espacio, mamar, comer, contacto madre hijo y comportamiento físico. En el segundo nivel, imagen corporal, coordinación de los dos lados del cuerpo (o praxia), nivel de actividad, atención, estabilidad emocional. En el tercer nivel, desarrolla habla y lenguaje, coordinación ojo-mano, percepción de forma y espacio y actividad con propósito. En el cuarto nivel y más completo se presentan los productos finales de los aprendizajes: habilidades para concentrarse y organizarse, obtener un buen concepto de sí mismo y control y confianza en sí mismo, capacidad de razonar y pensar de manera abstracta, especialización de un lado del cuerpo y de un lado de cerebro. 


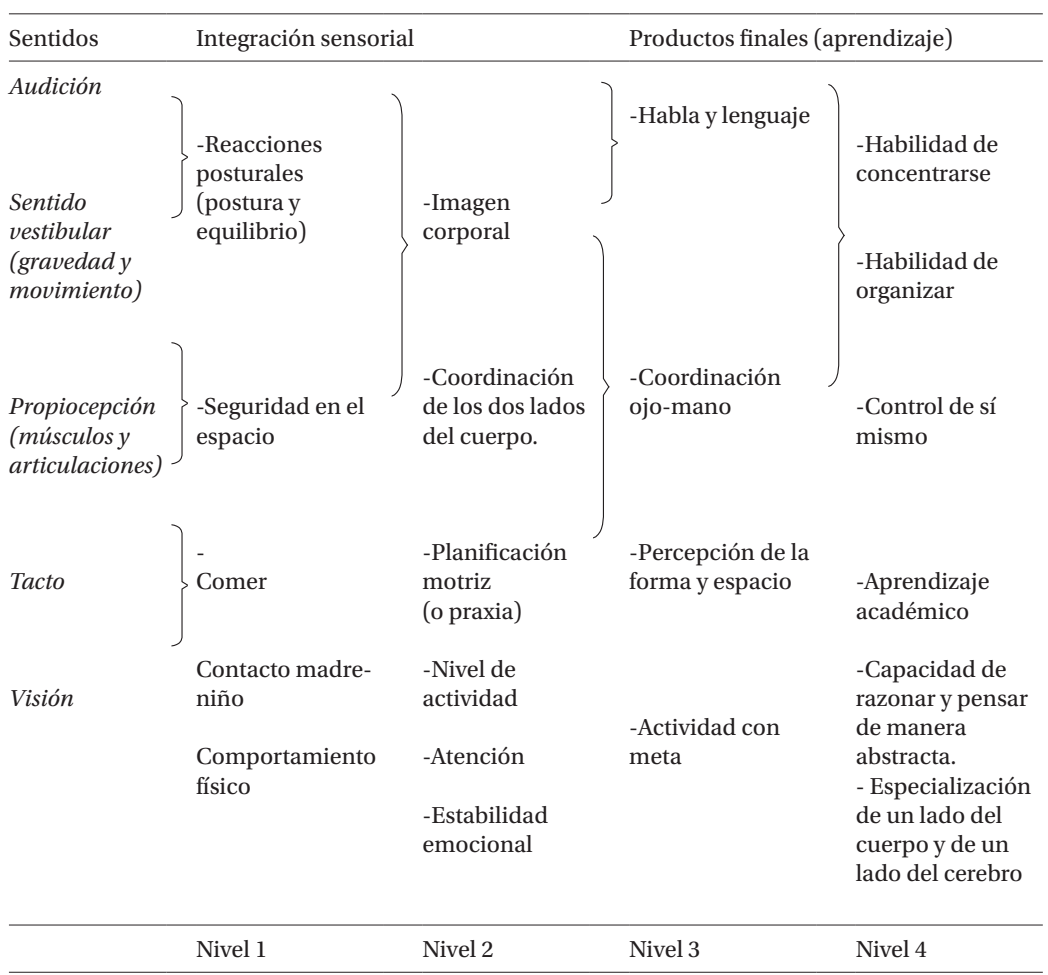

Gráfica 1. Resumen sinóptico del desarrollo integral, elaborado por la Dra. Jean Ayres

Fuente: Integración sensorial, Ayres, 2006.

En el proceso sensorial, como muestra la gráfica 1, la base de todo la establecen los sentidos. Estos permiten el desarrollo de habilidades, como el planeamiento motor, el reconocimiento y construcción del esquema corporal, la seguridad postural y la conciencia de los dos lados del cuerpo. Con estos elementos se logra mejor coordinación óculomanual, habilidades auditivas y lenguaje, motricidad gruesa y atención. La integración sensorial aporta a un mejor aprendizaje y comportamiento, y el niño puede enfrentar de mejor forma las actividades de la vida diaria.

\section{Pedagogía sensorial}

La pedagogía sensorial tiene en cuenta que es a través de los sentidos como los niños y niñas inician su interacción con el mundo que le rodea, ya que a partir de estas vivencias construyen nuevos conceptos. 
En la educación inicial, por tanto, se hace necesario brindar diversas experiencias: "El educador está obligado a poner mayor empeño en la presentación ordenada y coordinada de todo lo que los alumnos pueden percibir hasta convertir la educación sensorial en la más importante del currículo educativo de este nivel" (Soler Fiérrez, 1992, p. 10).

Diversos autores han abordado el tema de la pedagogía sensorial y su importancia, entre ellos podemos nombrar a María Montessori (1870-1952), que estudió medicina y luego inició un trabajo con niños en una clínica especializada en neuropsiquiatría, con niños que para la época eran catalogados como deficientes. Mediante la aplicación del método científico y teniendo en cuenta las necesidades de la población de esta clínica, desarrolla recursos didácticos de su propia autoría e ingenio, innovadores para este momento en la historia de la pedagogía. Entre estos incluye algunos específicamente sensoriales, buscando potenciar las habilidades de este grupo.

Es fundamental que la pedagogía preescolar implique la estimulación de los sentidos, comprendiendo que esta es la forma en la que los niños desarrollan un aprendizaje adecuado: "No se puede seguir considerando a la educación sensorial como algo espontáneo, fruto exclusivo de los procesos de maduración, sino que depende muy fundamentalmente de la experiencia y del aprendizaje" (García Hoz, 1996, p. 45).

\section{Metodología}

De acuerdo con McMillan (2005, p. 16), "el proceso de investigación incluye, normalmente, varias fases. Estas no son secuenciales ni constituyen un proceso ordenado paso a paso. La investigación es un proceso interactivo entre el investigador y la lógica del problema, el diseño y la interpretación". La presente investigación se desarrolla con un enfoque mixto, como investigación-acción.

La finalidad de este estudio es analizar los resultados encontrados en la implementación de la integración sensorial como estrategia-lúdica, especialmente diseñada para favorecer el desarrollo integral de niños y niñas. Se desarrolla con una población de niños y niñas de entre los 4 y 6 años, de un jardín infantil privado, el Globito Rojo y de una institución pública, la Escuela Normal Superior Distrital María Mon- 
tessori de la ciudad de Bogotá. Se contó con el consentimiento informado de los padres de familia.

Las actividades de los talleres están enfocadas en la estimulación de los sistemas sensoriales, principalmente los sistemas táctil, vestibular y propioceptivo. De igual manera, también se mejoran las prácticas pedagógicas del docente, que puede ayudar en las transformaciones desde las instituciones educativas de primera infancia, como lo plantea la investigación acción educativa.

En cuanto a la recolección de datos, se realiza, en primer lugar, el procedimiento de obtención del consentimiento informado a los padres de familia, cumpliendo con los aspectos señalados en los artículos 15 y 16, de la Resolución 08430 de 1993, del Ministerio de Salud.

Como instrumento de recolección de datos se recurre a la "Escala Abreviada de Desarrollo" EAD-1 (Ministerio de Salud \& Unicef, 1999), que proporciona un método estándar para medir el desarrollo y detectar tempranamente a los niños de mayor riesgo en edades de 4 a 6 años. Con este instrumento se recolecta y procesa la información, la cual permite evidenciar las dificultades individuales y, a su vez, dar a conocer la dificultad grupal. De acuerdo con lo mencionado, se analizan, en la escala abreviada de desarrollo, motricidad gruesa, motricidad fino-adaptativa, audición lenguaje y personal social.

Asimismo, se diseña, elabora e implementa en la propuesta 10 talleres, realizados en una temporalidad semanal por parte de las investigadoras, buscando promover estímulos sensoriales enfocados en la estimulación de los sentidos táctil, vestibular y propioceptivo, que son parte de la teoría de la integración sensorial, los cuales corresponden a: 1. Diversión y movimiento, 2. Tocamos, exploramos y sentimos, 3 . Circuito de superfuertes, 4 . El placer de sentir y oler, 5 . Movimiento y diversión, 6. Retos divertidos, 7. Soplando y jugando, 8. Diversas sensaciones en mi piel, 9. Escuchando y jugando con "Mariana y el Campamento", y 10. Compartiendo y jugando.

Se analiza la información obtenida, organizando los datos recolectados en una matriz elaborada en Microsoft-Excel ${ }^{\circ}$, teniendo en cuenta el pretest y el postest, las anotaciones de cada una sesiones de talleres, ficha de chequeo e igualmente registros fotográficos de cada sesión por separado de cada una de las instituciones. 


\section{Producción de información}

Escala de abreviada de desarrollo: Se utilizó como pretest, al inicio del año lectivo 2018, y como postest al finalizar los talleres propuestos, y permitió valorar y obtener la información del estado inicial, correspondiente al desarrollo de los niños de 37 a 72 meses, así como evidenciar los avances en el desarrollo posterior a la implementación de los talleres, identificando sus avances, fortalezas y debilidades, lo que posibilitó detectar tempranamente alteraciones que se manifestaban y prevenirlas oportunamente (Ministerio de Salud, 1999).

Los ítems generales que se evalúan en la escala abreviada, de acuerdo con el área, son:

- Motricidad gruesa

- Motricidad fino-adaptativa

- Audición y lenguaje

- Personal social

Talleres de integración sensorial: Teniendo en cuenta la relación de la integración sensorial con el desarrollo integral, en los diferentes talleres se realizó una intervención con temporalidad semanal, en los que se asoció los sistemas táctil, vestibular y propioceptivo de la siguiente forma:

- Talleres 1, 3, 5, 6, 7, 10: Sistemas vestibular y propioceptivo, que permiten a los estudiantes fortalecer sus habilidades para concentrarse, habilidad para organizar, autorregulación, especialización para cada lado del cuerpo y del cerebro, habilidad para el aprendizaje académico.

- Talleres 2, 4, 8: Sistema táctil, que permite a los estudiantes favorecer sus habilidades para discriminar olor, forma y tamaño; para discriminar temperatura y textura, fomentar el aprendizaje con información sensorial y táctil, desarrollar la creatividad por medio de experiencias tangibles y concretas, manos y dedos fuertes para agarrar, apretar, pellizcar y sostener; fomentar el aprendizaje con información sensorial, olfativa y táctil, estimulación de la memoria olfativa.

- Taller 9: Sistemas auditivo, táctil, vestibular y propioceptivo, los que permiten a los estudiantes favorecer sus habilidades de escucha, de 
atención, seguimiento viso motor; fomentar el aprendizaje con información sensorial y táctil.

Ficha de chequeo: Facilita la recogida y análisis de cada taller mediante la observación directa, apoyada posteriormente por evidencias registradas en fotografías, para describir los momentos y alcances de cada sesión.

\section{Resultados}

Los resultados obtenidos en el pre y postest de la escala abreviada de desarrollo, de acuerdo con las categorías motricidad gruesa, motricidad fina adaptativa, audición-lenguaje y personal social, y de los 36 ítems por los que está conformada cada una, fueron los siguientes. El criterio de alerta indica situaciones de riesgo en el desarrollo, medio, que hay aspectos por mejorar, medio alto, que el estudiante va en un proceso de mejora, y alto significa que, de acuerdo con la valoración realizada, no se presentan dificultades en el desarrollo.

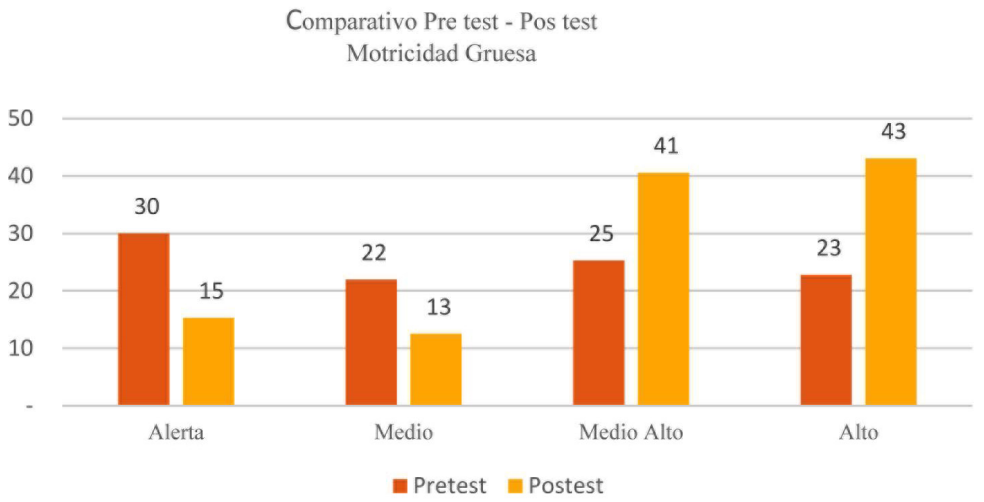

Gráfica 2. Comparativo entre pre y postest Motricidad gruesa Fuente: Elaboración propia 2020.

El siguiente análisis corresponde al comparativo realizado en la categoría "motricidad gruesa" entre el pre y el postest, en el que se evidencia que, en los diferentes ítems por los que está conformada la escala abreviada del desarrollo en esta categoría, aún no se logró un avance tan significativo como se esperaba, ya que solo un $43 \%$ de los estudiantes obtuvieron un nivel alto, en comparación a los resultados 
iniciales que eran de $23 \%$ para esta misma categoría. Sin embargo, las demás franjas mejoraron en comparación con el pretest inicial.

Comparativo Pre test - Pos test

Motricidad Fina Adaptativa

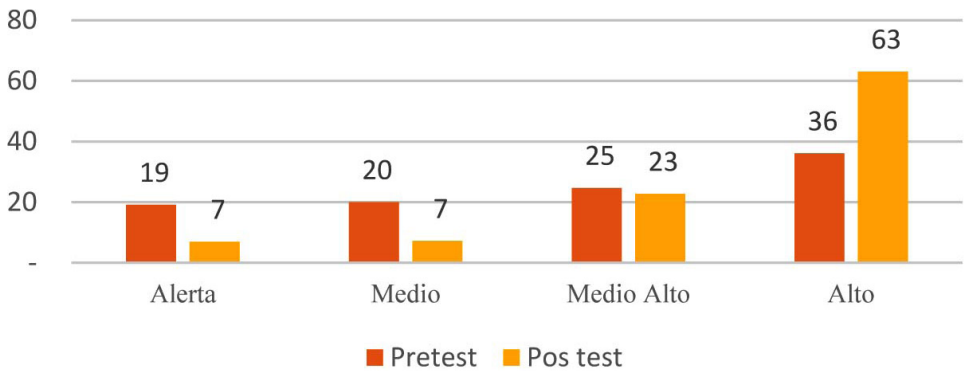

Gráfica 3. Comparativo entre pre y postest Motricidad Fina Adaptativa Fuente: Elaboración propia 2020.

De acuerdo con la gráfica de pretest y postest en la categoría de "motricidad fina adaptativa" de la escala abreviada de desarrollo, los avances en los estudiantes fueron bastante considerables, alcanzando en los diferentes ítems en alto un $63 \%$, lo que es de gran impacto, disminuyendo en el postest en las demás franjas.

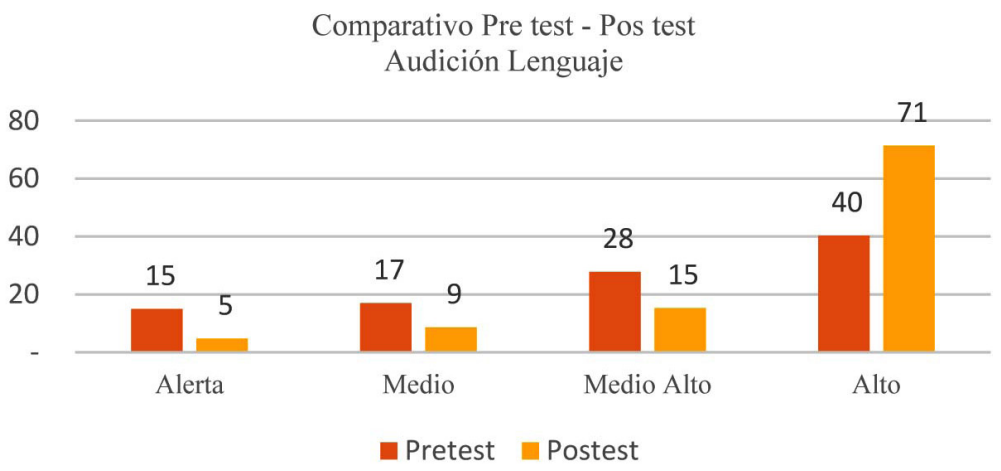

Gráfica 4. Comparativo entre pre y postest Audición Lenguaje Fuente: Elaboración propia 2020. 
Con base en la gráfica de pre y postest en la categoría de "audición lenguaje" de la escala abreviada de desarrollo, los estudiantes lograron un porcentaje en la franja de alto del $71 \%$, lo cual fue muy satisfactorio, ya que las intervenciones del taller aportaron en esta dimensión en cada uno. De igual manera, en el postest se disminuyó en las demás franjas.

\section{Comparativo Pre test - Pos test \\ Personal Social}

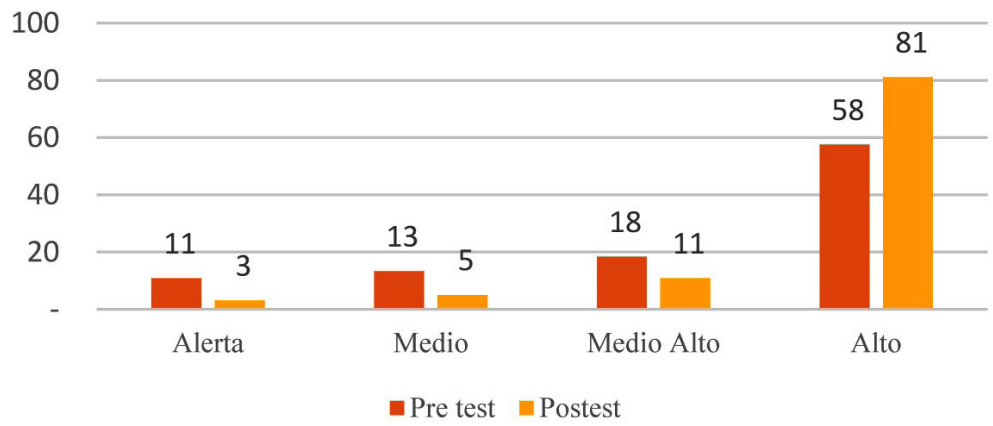

Gráfica 5. Comparativo entre pre y postest Personal Social Fuente: Elaboración propia 2020.

Con relación a los avances en la categoría "personal social" del pretest y postest de la escala abreviada de desarrollo, es evidente que, en la franja alto, correspondiente al $81 \%$ de los estudiantes, después de la implementación de los talleres mejoró significativamente y evidenció los avances que lograron los niños y niñas de 4 a 6 años.

Tabla 2

Consolidado del análisis de los talleres de integración sensorial

\begin{tabular}{lclc}
\hline & Porcentaje & & Frecuencia \\
\hline Vestibular & 10,75 & 89,25 & 43 \\
\hline Propioceptivo & 19,25 & 80,75 & 77 \\
\hline Táctil & 4,5 & 95,5 & 18 \\
\hline
\end{tabular}

Fuente: Elaboración propia 2020. 
La realización de los talleres de integración sensorial fue oportuna, evidenciando la motivación y participación de los estudiantes. De igual manera, transformar las prácticas pedagógicas e incorporar estrategias que no son de la cotidianidad del aula, ayudó a los estudiantes en su desarrollo y dio a las investigadoras herramientas muevas e importantes para el trabajo pedagógico de manera lúdica.

Como se observa en la gráfica, de acuerdo con la teoría de integración sensorial, en los estudiantes favoreció la información que los estudiantes reciben para organizarla, procesarla de forma indirecta y dar una respuesta sensorial. Los estudiantes mostraron un procesamiento sensorial adecuado en el sistema táctil, con un 95\%; en el sistema propioceptivo, con el $80 \%$, y en el sistema vestibular, con un $89 \%$, al participar en las intervenciones mediadas por los talleres de integración sensorial.

También se observó dificultades, en menor grado, en el procesamiento sensorial y respuesta adecuados. En el sistema táctil con un $4,5 \%$, en el propioceptivo con un $19,25 \%$ y en el vestibular con un $10,75 \%$.

\section{Discusión y conclusiones}

Es determinante la teoría de integración sensorial y los aportes que puede realizar a la educación para la primera infancia; sin embargo, se requiere cada vez mayor investigación al respecto; igualmente, que se establezca mayor interés por parte de los docentes en cuanto al tema. En este sentido, se requiere de capacitaciones que brinden mayor entendimiento y comprensión, no para que el docente realice el trabajo de un terapeuta, sino más bien para que se apropie de estrategias, actividades, conceptos teóricos, entre otros, que pueden ser parte de las practicas pedagógicas. Un aspecto que a las autoras de esta investigación les queda como experiencia es cómo convertir nuestra sala de clase en lugar de innovación pedagógica, donde se pueda aprender jugando, pero también buscando el desarrollo de los niños y niñas desde un enfoque lúdico, experimental y progresista.

Esta investigación es una contribución para ver con otros ojos la educación e, igualmente, para entender que conocer los procesos neurológicos, el funcionamiento del cerebro y los procesos de integración 
sensorial es necesario en la formación y actualización de los docentes, porque son aspectos fundamentales para un pleno desarrollo cognitivo, comunicativo, corporal y social de los estudiantes.

Asimismo, se establece la importancia de la estimulación sensorial para el desarrollo de los niños y niñas de 4 a 6 años, ya que en esta etapa se cuenta con un mapa mental dinámico y plástico, denominándose esta etapa como "periodos sensibles" o "ventanas de oportunidad", establecidas hasta los ocho años según los estudios (Díaz, 2013).

En la investigación se deja claro que el desarrollo de estrategias lúdicas de integración sensorial es de gran beneficio para cada uno de los niños y niñas, fortaleciendo su desarrollo, logrando una reducción en las alertas de motricidad fina adaptativa, audición-lenguaje y personal social. Asimismo, en los resultados de la aplicación de la escala se observó un aumento de estudiantes que alcanzaron un nivel alto, con posterioridad a la implementación del taller. De acuerdo con lo anterior, podemos ver que, en estos tres aspectos evaluados en la escala abreviada del desarrollo, los alcances fueron positivos para el desarrollo de los niños y niñas, como se planteó inicialmente.

Como demuestran los resultados de la investigación, respecto de la dimensión de motricidad gruesa, se lograron avances; sin embargo, se evidencia cómo, en este aspecto, se mantienen niños en alerta, medio y medio alto, y es menor el número de estudiantes que alcanzan nivel alto, posterior a la implantación de los talleres. En consecuencia, es importante resaltar que, en este aspecto evaluado, como es la motricidad gruesa, es necesario continuar trabajando y estimulando sensorialmente, mediante diferentes actividades y estrategias, a los niños y niñas, ya que esta dimensión va ligada al desarrollo cognitivo.

Los resultados globales de los grupos en los que se implementaron los talleres lúdicos de integración sensorial, que atendían a las necesidades evidenciadas en los niños y niñas, contribuyeron al fortalecimiento de los procesos de desarrollo, respondiendo adecuadamente a los estímulos, en el sistema táctil con un 95\%, en el sistema propioceptivo con un $80 \%$ y en el sistema vestibular con un $89 \%$.

Se hizo patente la motivación e implicación de los estudiantes, ya los recursos didácticos y actividades realizadas en el transcurso de los talleres fueron del goce y el disfrute para los niños y niñas. y así se in- 
volucraron con mayor entusiasmo, solicitando que se realizaran de nuevo, puesto que se divertían mucho con la utilización de los múltiples elementos, por su representación en colores, formas, tamaños, olores, texturas. Los retos propuestos en los talleres permitieron que, gradualmente, los estudiantes fueran adquiriendo y potenciando sus habilidades con relación la integración sensorial, y para favorecer el desarrollo adecuado de los niños y niñas de los 4 a 6 años. De igual forma, se encontró algunos niños en alerta, de acuerdo con la escala abreviada de desarrollo, detectando a tiempo factores de riesgo. Estos estudiantes fueron remitidos para apoyo interdisciplinario, según su necesidad.

Los talleres de integración sensorial permitieron llevar al aula de clase una didáctica que enriqueció las prácticas pedagógicas, cambiando la manera de entender, enseñar y, a su vez, la forma en que los estudiantes aprendían, debido a que generó más conocimiento a las investigadoras para realizar sus clases con materiales diversos, lo que a su vez produjo en los estudiantes alegría y entusiasmo por el aprendizaje, y un mejor desarrollo de una manera lúdica.

En el hallazgo de la temática relacionada con integración sensorial se encontró que existe poca literatura en idioma español, ya que la mayoría se encuentra en inglés. De igual manera, las investigaciones de integración sensorial con relación al desarrollo infantil aplicado a la educación son muy escasas, porque en su mayoría se enfocan en el trabajo desarrollado por terapeutas ocupacionales.

Asimismo, respecto del marco teórico, son pocas las investigaciones acerca de la relación entre el desarrollo de niños y niñas y la integración sensorial enfocada a la educación en el aula de clase. Por tal motivo, la investigación puede continuar su desarrollo enfocándose en la pedagogía del aula de clase y proyectándose a la interdisciplinariedad, con los profesionales de la salud ocupacional, para formular nuevas hipótesis de investigación.

Otra posibilidad para lograr la escalabilidad de la investigación es indagar en nuevos grupos de niños y niñas con diferentes condiciones socioeconómicas, raciales y espaciales, de modo de analizar los resultados al aplicar las estrategias de integración sensorial en distintas poblaciones de estudio. De igual manera, es interesante a futuro 
analizar la incidencia en el desarrollo de los niños y niñas, de aulas de integración sensorial equipadas con elementos lúdicos diseñados para este fin y ubicadas de manera regular en colegios privados y públicos de Colombia.

A partir de esta investigación se constató la importancia de involucrar la pedagogía sensorial en el aula de clase, al igual que los fundamentos neurológicos que trabaja la integración sensorial para favorecer el desarrollo de niños y niñas.

Se logra contribuir en la innovación en los espacios pedagógicos de la primera infancia, por medio de la IS como estrategia lúdica, ya que el juego es parte fundamental en la forma de aprender y es un pilar de la educación colombiana. Teniendo en cuenta también que las actividades y materiales usados en IS están planteados para que niños y niñas se diviertan y aprendan al mismo tiempo.

De igual manera, se proponen cambios en las prácticas educativas, ya que el papel de los docentes es cada vez más complejo y demanda la inclusión de estudiantes con necesidades diversas, esperando que en las aulas se desarrollen estrategias innovadoras que busquen y garanticen que todos los niños y niñas tengan un desarrollo integral.

Durante la investigación, fue innovador utilizar una teoría implementada por terapeutas ocupacionales para la intervención y apoyo en diferentes dificultades del desarrollo. En nuestra intervención se realizó una apropiación y aprovechamiento de aspectos teóricos, prácticos y de recursos abordados por los terapeutas de integración sensorial, incorporándolos en las prácticas pedagógicas, con el fin de que, desde el aula de clase, se aporte al desarrollo integral como parte de la prevención y detección de dificultades en los niños de 4 a 6 años.

A partir de la investigación se logró realizar aportes a los padres de familia, al utilizar recursos que sirven de apoyo a sus hijos en diferentes aspectos del desarrollo, por medio de la integración sensorial.

Gracias a teoría de integración sensorial y a la práctica realizada, se observó que muchos de los recursos y materiales del aula de clase, entre ellos material reciclable, como cubetas de huevo, telas, tapas de gaseosa, entre otros, sirven para el beneficio del aprendizaje y el desarrollo. De igual manera, algunos materiales especiales de integración sensorial son un poco más costosos y no se tienen en las institucio- 
nes, ni los espacios son los indicados para tener un aula multisensorial. Aunque en los talleres de integración sensorial se usaron diversas estrategias, es evidente que faltaron más de la misma teoría, las cuales aportan para el desarrollo y el aprendizaje de niñas y niños de manera lúdica.

\section{Referencias bibliográficas}

Alves, K. (2017). Integración Sensorial: la importancia de sentir. Instituto Superior de Estudios Psicológicos. Recuperado de https:// www.isep.es/actualidad-neurociencias/integracion-sensorial-la-importancia-de-sentir/

Ayres, J. (1998). Integración sensorial y el niño. México: Trillas.

Ayres, J. (2006). La integración sensorial y el niño. México: Trillas.

Beaudry, I. (2003). Los problemas de aprendizaje en la infancia. Oviedo: Nobel.

Bialer, D. \& Miller, L. J. (2011). No longer a secret: unique common sense strategies for children with sensory or motor challenges. London: Eurospan distributor.

Camacaro, M. (2013). Estrategias para el abordaje educativo del sentido táctil en la educación física infantil. Revista de Investigación, 93.

Díaz, M. G. (2013). Neurociencias y educación. Chile: Mago Editores.

García Hoz, V. (1996). Educación infantil personalizada. Madrid: Rialp.

García, V. (1996). Educación infantil personalizada. Madrid: Rialp.

Gonzáles, J. (2006). Perspectivas contemporáneas sobre la cognición, categorización, conceptualización. Argentina: Siglo XXI Editores.

Lazáro, A. (2008). Estimulación vestibular en educación infantil. Revista Interuniversitaria de Formación del Profesorado, 172.

Martínez, E. J. (2014). Desarrollo psicomotor en educación infantil. Almeira: Universidad de Almeira.

McMillan, J. y Schumacher, S. (2005). Investigación educativa $5^{a}$ edición. Madrid: Pearson Educación.

Miller, L. y Bialer, D. (2012). No longer a secret. London: EuroSpan distributor.

Ministerio de Educación Nacional. (26 de mayo de 2015). Decreto 1075, Por medio del cual se expide el Decreto Único Reglamentario 
del Sector Educación. Recuperado de http://redes.colombiaaprende.edu.co/ntg/men/pdf/decreto_1075_de_2015.pdf

Ministerio de Salud. (1999). Escala abreviada de desarrollo. Bogotá.

Navas, D. C. (2011). Desarrollo cognitivo, sensorial, motor y psicomotor en la infancia. Málaga: Innovación y Cualificación.

Sánchez, E. (2010). Bases neurológicas y psicopedagógicas del tratamiento educativo de la diversidad. Madrid: Universidad Nacional de Educación a Distancia.

Senkow, A. (2018). Unlocking behavior: interventions for children with sensory processing disorder. California State University San Marcos. Recuperado el 27 de junio de 2018, de http://csusmdspace.calstate.edu/bitstream/handle/10211.3/202824/ SenkowApril_Spring\%202018.pdf?sequence $=3$

Soler Fiérrez, E. (1992). La educación sensorial en la escuela infantil. Madrid: Rialp.

Stock, C. (1998). 101 actividades para entretener a tu hijo en lugares cerrados. Barcelona: Paidós.

Thoumi, S. (2003). Técnicas de la motivación infantil. Colombia: Gamma.

Yack, E., Sutton, S. \& P. Aquilla. (2002). Building Bridges Through Sensory Integration. Arlington: Future Horizons. 